\title{
CONGRUENCES BETWEEN ABELIAN PSEUDOMEASURES
}

\author{
Jürgen Ritter And Alfred Weiss \\ Dedicated to Professor Peter Roquette on his $80^{\text {th }}$ birthday
}

In this paper $K$ is a totally real number field (finite over $\mathbb{Q}$ ), $p$ a fixed odd prime number, and $S$ a fixed finite set of non-archimedian primes of $K$ containing all primes above $p$. Let $K_{S}$ denote the maximal abelian extension of $K$ which is unramified (at all non-archimedean primes) outside $S$ and set $G_{S}=G\left(K_{S} / K\right)$. Serre's pseudomeasure $\lambda_{K}=\lambda_{K, S}$ has the property that $(1-g) \lambda_{K}$ is in the completed group ring $\mathbb{Z}_{p}\left[\left[G_{S}\right]\right]$ for all $g \in G_{S}[\mathrm{Se} 2]$.

Let $L$ be a totally real Galois extension of $K$ of degree $p$ with group $\Sigma=G(L / K)$. Moreover, assume that the finite set $S$ of places contains all the primes of $K$ which ramify in $L$. Let $L_{S}, H_{S}=G\left(L_{S} / L\right)$ and $\lambda_{L}$ be the corresponding objects over $L$ with respect to the set of primes of $L$ above $S$. Observe that $L_{S}$ is a Galois extension of $K$, with group $\mathfrak{G}$, hence $G_{S}$ is its maximal abelian factor group. Also, since $H_{S}$ is a normal subgroup of $\mathfrak{G}$ of index $p$, the quotient $\Sigma=\mathfrak{G} / H_{S}$ acts on $H_{S}$ by conjugation. This situation induces the transfer map ver : $G_{S} \rightarrow H_{S}$ by means of which we can compare $\lambda_{K}$ and $\lambda_{L}$.

Theorem.: For $g_{K} \in G_{S}$ and $h_{L}=\operatorname{ver}\left(g_{K}\right) \in H_{S}$,

$$
\operatorname{ver}\left(\lambda_{g_{K}}\right) \equiv \lambda_{h_{L}} \quad \bmod T,
$$

where $\lambda_{g_{K}}=\left(1-g_{K}\right) \lambda_{K, S}, \lambda_{h_{L}}=\left(1-h_{L}\right) \lambda_{L, S}$, and where $T$ is the ideal in the ring $\mathbb{Z}_{p}\left[\left[H_{S}\right]\right]^{\Sigma}$ of $\Sigma$-fixed points of $\mathbb{Z}_{p}\left[\left[H_{S}\right]\right]$ consisting of all $\Sigma$-traces $\sum_{\sigma \in \Sigma} \alpha^{\sigma}, \alpha \in \mathbb{Z}_{p}\left[\left[H_{S}\right]\right]$.

The proof follows from Deligne and Ribet [DR] by interpreting it on the Galois side as in [Se2]. Explicitly, the group $\lim _{\leftarrow} G_{f}$ of [DR, p.230], with $\mathfrak{f}$ running through the integral ideals of $K$ with all prime factors in $S$, is identified with our $G_{S}$, via class field theory.

The theorem implies the "torsion congruences" of [RW, §3] in general, and thus the proof of the 'main conjecture of equivariant Iwasawa theory' of [RW2, §4 $]^{1}$ is reduced to proving the integrality of the logarithmic pseudomeasure $t$ of [RW, 22$]$.

More precisely, let $L_{\infty}$ be the cyclotomic $\mathbb{Z}_{p}$-extension of $L$ and $\Gamma_{L}=G\left(L_{\infty} / L\right)$. If the element $g_{K} \in G_{S}$ (as above) has infinite order, then the image of $\left(1-h_{L}\right)$ under $\mathbb{Z}_{p}\left[\left[H_{S}\right]\right] \rightarrow \mathbb{Z}_{p}\left[\left[\Gamma_{L}\right]\right]$ is not in $p \mathbb{Z}_{p}\left[\left[\Gamma_{L}\right]\right]$. Letting $\mathbb{Z}_{p}\left[\left[H_{S}\right]\right]$. be the localization obtained

Received by the editors July 10, 2007.

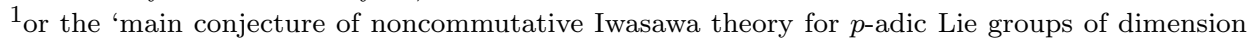
$1 '($ see $[K, \S 3])$ 
by inverting the multiplicative set of elements of $\mathbb{Z}_{p}\left[\left[H_{S}\right]\right]$ whose image in $\mathbb{Z}_{p}\left[\left[\Gamma_{L}\right]\right]$ is not in $p \mathbb{Z}_{p}\left[\left[\Gamma_{L}\right]\right]$, the theorem reads

$$
\operatorname{ver}\left(\lambda_{K, S}\right) \equiv \lambda_{L, S} \quad \bmod T_{\bullet}
$$

with $T_{\bullet}$ the $\Sigma$-trace ideal in $\mathbb{Z}_{p}\left[\left[H_{S}\right]\right]_{\bullet}^{\Sigma}$. If $M$ is totally real and Galois over $K$ with $L_{\infty} \subset M \subset L_{S}$ and $\left[M: L_{\infty}\right]$ finite, then the "torsion congruence" is obtained by specializing $\mathfrak{G} \rightarrow G(M / K)$. Moreover, for $p$-extensions $M / K$ the "torsion congruences" also imply the "logarithmic congruences" of $[\mathrm{RW}]$, so that we get a proof of many cases of the "main conjecture" of [RW2,FK] complementing the Heisenberg extensions of Kato ${ }^{2}$.

Here is a short description of the individual sections to follow. In $\S 1$ we write $\lambda_{g_{K}}$ as a limit element in $\lim _{\overleftarrow{U}} \mathbb{Z}_{p}\left[G_{S} / U\right] / p^{m(U)}$ with $U$ open in $G_{S}$ and with certain integers $m(U)$. This allows us to study the claimed congruence on finite level, which is carried out in $\S 2$. The next section is some preparation concerning Hilbert modular forms that we need for the proof of the theorem, which in $\S 4$ is combined with the work of Deligne and Ribet to finish the proof. A final section briefly discusses a weaker version of the theorem when $p=2$.

\section{Approximations to pseudomeasures}

We review the construction of pseudomeasures [Se2] in a more explicit form that will be essential for our purposes. We first fix notation.

For a coset $x$ of an open subgroup $U$ of $G_{S}$ set $\delta^{(x)}(g)=1$ or 0 according to the cases $g \in x$ or $g \notin x$. Then, for integers $k \geq 1$, define $\zeta_{K}\left(1-k, \delta^{(x)}\right)=\zeta_{K, S}\left(1-k, \delta^{(x)}\right) \in \mathbb{Q}$ to be the value at $1-k$ of the partial $\zeta$-function for the set of integral ideals $\mathfrak{a}$ of $K$ prime to $S$ with Artin symbol $g_{\mathfrak{a}}$ in $x^{3}$. Note that the definition of $\zeta_{K}\left(1-k, \delta^{(x)}\right)$ extends linearly to locally constant functions $\varepsilon$ on $G_{S}$ with values in a $\mathbb{Q}$-vector space and gives values $\zeta_{K}(1-k, \varepsilon)$ in that vector space.

Let $\mathcal{N}=\mathcal{N}_{K, p}: G_{S} \rightarrow \mathbb{Z}_{p}{ }^{\times}$be the continuous character whose value on $g_{\mathfrak{a}}$ for an integral ideal $\mathfrak{a}$ prime to $S$ is its absolute norm $\mathcal{N} \mathfrak{a}{ }^{4}$. For $g \in G_{S}, k \geq 1$ and $\varepsilon$ a locally constant $\mathbb{Q}_{p}$-valued function on $G_{S}$ we define, following [DR],

$$
\Delta_{g}(1-k, \varepsilon)=\zeta_{K}(1-k, \varepsilon)-\mathcal{N}(g)^{k} \zeta_{K}\left(1-k, \varepsilon_{g}\right) \in \mathbb{Q}_{p},
$$

where $\varepsilon_{g}\left(g^{\prime}\right)=\varepsilon\left(g g^{\prime}\right)$ for $g^{\prime} \in G_{S}$.

We can now state

TheOREM [(0.4) of [DR]].: Let $\varepsilon_{1}, \varepsilon_{2}, \ldots$ be a finite sequence of locally constant functions $G_{S} \rightarrow \mathbb{Q}_{p}$ so that $\sum_{k \geq 1} \varepsilon_{k}\left(g^{\prime}\right) \mathcal{N}\left(g^{\prime}\right)^{k-1} \in \mathbb{Z}_{p}$ for all $g^{\prime} \in G_{S}$. Then

$$
\sum_{k \geq 1} \Delta_{g}\left(1-k, \varepsilon_{k}\right) \in \mathbb{Z}_{p} \quad \text { for all } g \in G_{S}
$$

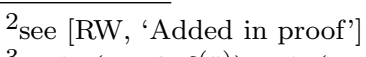

$3_{\text {So }} \zeta_{K}\left(1-k, \delta^{(x)}\right)=\zeta_{S}(x, 1-k)$ in [Se2], and $=L\left(1-k, \delta^{(x)}\right)$ in [DR], up to identification

$4_{\text {i.e., }} \mathcal{N}_{p}$ is the cyclotomic character, so $\mathcal{N}_{p}(g)$ is determined by the action of $g$ on $p$-power roots of unity (see [Se2, (2.3)]
} 
Call an open subgroup $U$ of $G_{S}$ admissible, if $\mathcal{N}(U) \subset 1+p \mathbb{Z}_{p}$, and define $m(U) \geq 1$ by $\mathcal{N}(U)=1+p^{m(U)} \mathbb{Z}_{p}$.

LEMMA 1.: If $U$ runs through the cofinal system of admissible open subgroups of $G_{S}$, then $\mathbb{Z}_{p}\left[\left[G_{S}\right]\right]=\lim _{\overleftarrow{U}} \mathbb{Z}_{p}\left[G_{S} / U\right] / p^{m(U)} \mathbb{Z}_{p}\left[G_{S} / U\right]$

Proof. The natural map

$$
\mathbb{Z}_{p}\left[\left[G_{S}\right]\right]=\lim _{\overleftarrow{U}} \mathbb{Z}_{p}\left[G_{S} / U\right] \rightarrow \lim _{\overleftarrow{U}} \mathbb{Z}_{p}\left[G_{S} / U\right] / p^{m(U)}
$$

is injective, since $m\left(U_{1}\right) \geq m\left(U_{2}\right)$ for $U_{1} \leq U_{2}$ and since the $m(U)$ 's are unbounded. In order to show surjectivity, it is sufficient to find a linearly ordered cofinal family $\left\{U^{\prime}\right\}$ of open subgroups, because then it follows that the image of $\underset{U^{\prime}}{\lim } \mathbb{Z}_{p}\left[G_{S} / U^{\prime}\right] / p^{m\left(U^{\prime}\right)}$ is dense in the compact group $\underset{U^{\prime}}{\lim } \mathbb{Z}_{p}\left[G_{S} / U^{\prime}\right] / p^{m\left(U^{\prime}\right)}$, by taking successive approximations which are compatible with the projections. Now, $G_{S}$ is finitely generated (over $\hat{\mathbb{Z}}$ ), as the inertia groups for the $\mathfrak{p} \in S$ are finitely generated and they together generate an open subgroup (the fixed field of which is the strict Hilbert class field of $K$ ). Thus $G_{S}$ is a homomorphic image of a finite product $\prod \hat{\mathbb{Z}}$ and hence the closed subgroup $\left(G_{S}\right)^{n !}$ has index dividing the finite order of $\prod(\hat{\mathbb{Z}} / n !)$ and so is open.

Proposition 2.: For $g \in G_{S}$ there is a unique element $\lambda_{g} \in \mathbb{Z}_{p}\left[\left[G_{S}\right]\right]$, independent of $k^{5}$, whose image in $\mathbb{Z}_{p}\left[G_{S} / U\right] / p^{m(U)}$ is

$$
\sum_{x \in G_{S} / U} \Delta_{g}\left(1-k, \delta^{(x)}\right) \mathcal{N}(x)^{-k} x \bmod p^{m(U)} \mathbb{Z}_{p}[G / U],
$$

for all admissible $U$, where $\mathcal{N}$ here also denotes the homomorphism $G_{S} / U \rightarrow$ $\left(\mathbb{Z}_{p} / p^{m(U)}\right)^{\times}$induced by our previous $\mathcal{N}$. Moreover, if $\lambda$ is the pseudomeasure of [Se2], then

$$
(1-g) \lambda=\lambda_{g}
$$

Note first that the displayed elements are well-defined by the definition of $m(U)$ and that, varying $U$, they determine a limit element $\lambda_{g} \in \mathbb{Z}_{p}\left[\left[G_{S}\right]\right]$, since $\Delta_{g}$ is a $\mathbb{Z}_{p^{-}}$-valued distribution (see $[\mathrm{DR},(0.5)]$ ).

We check that $\lambda_{g}$ is independent of $k$. Fix $U$ and a coset $x$. Choose a (set) map $\eta: G_{S} / U \rightarrow \mathbb{Z}_{p}{ }^{\times}$so that $\eta\left(g^{\prime} U\right) \equiv \mathcal{N}^{k-1}\left(g^{\prime}\right) \bmod p^{m(U)}$ for all $g^{\prime} \in G_{S}$. Viewing $\eta$ as a locally constant function on $G_{S}$, then

$$
\Delta_{g}\left(0, \delta^{(x)} \eta\right) \equiv \Delta_{g}\left(1-k, \delta^{(x)}\right) \bmod p^{m(U)} .
$$

To see this, apply Theorem [(0.4) of [DR]], repeated above, with $\varepsilon_{1}=p^{-m(U)} \delta^{(x)} \eta$, $\varepsilon_{k}=-p^{-m(U)} \delta^{(x)}$ ( and the other $\varepsilon$ 's zero). Hence, with $\tilde{x} \in x$,

$$
\begin{aligned}
& \Delta_{g}\left(1-k, \delta^{(x)}\right) \mathcal{N} \tilde{x}^{-k} \equiv \Delta_{g}\left(0, \delta^{(x)} \eta\right) \eta(x)^{-1} \mathcal{N} \tilde{x}^{-1} \\
& =\Delta_{g}\left(0, \eta(x)^{-1} \delta^{(x)} \eta\right) \mathcal{N} \tilde{x}^{-1}=\Delta_{g}\left(0, \delta^{(x)}\right) \mathcal{N} \tilde{x}^{-1} \bmod p^{m(U)} .
\end{aligned}
$$

We next check that our $\lambda_{g}$ satisfies

$$
\left\langle\varepsilon \mathcal{N}^{k}, \lambda_{g}\right\rangle=\Delta_{g}(1-k, \varepsilon)
$$

\footnotetext{
${ }^{5}$ This allows us to take $k>2$ to avoid difficulties with $K=\mathbb{Q}$.
} 
(compare $[\operatorname{Se} 2,(3.6)])$. As above, choose $\eta: G_{S} / U \rightarrow \mathbb{Z}_{p}{ }^{\times}$so that now $\eta(y U) \equiv \mathcal{N}^{k} y$ $\bmod p^{m(U)}$. Then, by $[\mathrm{Se} 2,(1.1)]$,

$$
\begin{aligned}
& \left\langle\varepsilon \mathcal{N}^{k}, \lambda_{g}\right\rangle \equiv\left\langle\varepsilon \eta, \lambda_{g}\right\rangle \equiv \sum_{x} \varepsilon \eta(x) \Delta_{g}\left(1-k, \delta^{(x)}\right) \mathcal{N} x^{-k} \\
& \equiv \sum_{x} \varepsilon(x) \Delta_{g}\left(1-k, \delta^{(x)}\right)=\Delta_{g}\left(1-k, \sum_{x} \varepsilon(x) \delta^{(x)}\right)=\Delta_{g}(1-k, \varepsilon) \quad \bmod p^{m(U)} .
\end{aligned}
$$

By the argument following [Se2, (3.6)] it follows that $(1-g) \lambda$ is equal to our $\lambda_{g}$ for all $g \in G_{S}$.

\section{TRANSFER}

Let $L / K$ be as in the introduction. We decorate objects which depend on $L$ and are analogous to the ones of $K$ appropriately, e.g. $\mathcal{N}_{L}, m_{L}, \ldots$; in particular we have the notion of admissible open subgroups of $H_{S}$. Note that if $V$ is such an admissible open subgroup of $H_{S}$, then $\bigcap_{\sigma \in \Sigma} V^{\sigma}$ is also open and therefore the system of $\Sigma$-stable admissible open subgroups of $H_{S}$ is a cofinal system of open subgroups of $H_{S}$.

LemMA 3.: (1) If $V$ is an admissible open subgroup of $H_{S}$ and $U$ is an admissible open subgroup of $G_{S}$ contained in $\operatorname{ver}^{-1}(V)$, then $m_{K}(U) \geq$ $m_{L}(V)-1$.

(2) Let $y$ be a coset of a $\Sigma$-stable admissible open subgroup of $H_{S}$. If $h \in H_{S}$ is fixed by $\Sigma$, then $\Delta_{h}\left(1-k, \delta_{L}^{\left(y^{\sigma}\right)}\right)=\Delta_{h}\left(1-k, \delta_{L}^{(y)}\right)$, where $\Delta_{h}=\Delta_{L, h}$. In particular, $\lambda_{h_{L}}$ is fixed by $\Sigma$.

The first assertion uses $\mathcal{N}_{L}(\operatorname{ver}(g))=\mathcal{N}_{K}(g)^{p}$ for $g \in G_{S}$. Now $U \leq \operatorname{ver}^{-1}(V)$ implies $\operatorname{ver}(U) \leq V$, hence $\mathcal{N}_{L}(V) \supset \mathcal{N}_{L}(\operatorname{ver}(U))=\mathcal{N}_{K}(U)^{p}$, i.e., $1+p^{m_{L}(V)} \mathbb{Z}_{p} \supset$ $\left(1+p^{m_{K}(U)} \mathbb{Z}_{p}\right)^{p}=1+p^{m_{K}(U)+1} \mathbb{Z}_{p}$. Thus $m_{K}(U)+1 \geq m_{L}(V)$.

For the second assertion it suffices to show that $\zeta_{L}\left(1-k, \delta_{L}^{\left(y^{\sigma}\right)}\right)=\zeta_{L}\left(1-k, \delta_{L}^{(y)}\right)$ for all $y$, because $\left(\delta_{L}^{\left(y^{\sigma}\right)}\right)_{h}=\delta_{L}^{\left(h^{-1} y^{\sigma}\right)}=\delta_{L}^{\left(\left(h^{-1} y\right)^{\sigma}\right)}$ and $\delta_{L}^{\left(h^{-1} y\right)}=\left(\delta_{L}^{(y)}\right)_{h}$. Now view $\delta_{L}^{(y)}$ as a complex valued function on $H_{S} / V$ and write it as a $\mathbb{C}$-linear combination of the (abelian) characters $\chi$ of $H_{S} / V$. It suffices to check whether $\zeta_{L}(1-k, \chi)=\zeta_{L}(1-$ $\left.k, \chi^{\sigma}\right)$, with $\chi^{\sigma}(h)=\chi\left(h^{\sigma^{-1}}\right)=\chi\left(\sigma h \sigma^{-1}\right)$. But this follows from the compatibility of the Artin $L$-functions with induction, because ind ${ }_{H_{S} / V}^{\mathfrak{G} / V} \chi=$ ind $_{H_{S} / V}^{\mathfrak{G} / V} \chi^{\sigma}$.

This finishes the proof.

Let $N$ be the kernel of ver : $G_{S} \rightarrow H_{S}$. A $\Sigma$-stable admissible open subgroup $V$ of $H_{S}$ gives rise to the transfer map $G_{S} / U \rightarrow H_{S} / V$ whenever $U \leq \operatorname{ver}^{-1}(V)$. These transfer maps combined yield the right hand map in the commutative square

$$
\begin{array}{llcc}
\mathbb{Z}_{p}\left[\left[G_{S}\right]\right] & \rightarrow & \lim _{U \succeq N} \mathbb{Z}_{p}\left[G_{S} / U\right] / p^{m_{K}(U)} \\
\text { ver } \downarrow & & & \downarrow \\
\mathbb{Z}_{p}\left[\left[H_{S}\right]\right] & \stackrel{\simeq}{\longrightarrow} & \lim _{V, \Sigma-\text { stable }} \mathbb{Z}_{p}\left[H_{S} / V\right] / p^{m_{L}(V)-1},
\end{array}
$$

explicitly sending $\left(x_{U}\right)_{U}$ to $\left(y_{V}\right)_{V}$ with $y_{V}$ the image of $x_{U}$ under $\mathbb{Z}_{p}\left[G_{S} / U\right] / p^{m_{K}(U)}$ $\stackrel{\text { ver }}{\longrightarrow} \mathbb{Z}_{l}\left[H_{S} / V\right] / p^{m_{K}(U)} \rightarrow \mathbb{Z}_{p}\left[H_{S} / V\right] / p^{m_{L}(V)-1}$ whenever $U \leq \operatorname{ver}^{-1}(V)$. The bottom arrow is an isomorphism by the proof of Lemma 1.

We recall that a locally constant function $\varepsilon_{L}$ on $H_{S}$ is even, if $\varepsilon_{L}\left(c_{w} h\right)=\varepsilon_{L}(h)$ for all $h \in H_{S}$ and all "Frobenius elements" $c_{w}$ at the archimedean primes $w$ of $L$ (so 
$c_{w} \in H_{S}$ is the restriction of complex conjugation with respect to an embedding $L_{S} \hookrightarrow \mathbb{C}$ inducing $w$ on $L$ ).

Set $\mathbb{Z}_{(p)}=\mathbb{Q} \cap \mathbb{Z}_{p}$.

Proposition 4.: A sufficient condition for the Theorem in the introduction to hold is the following:

$$
\Delta_{h_{L}}\left(1-k, \varepsilon_{L}\right) \equiv \Delta_{g_{K}}\left(1-p k, \varepsilon_{L} \circ \text { ver }\right) \bmod p \mathbb{Z}_{p}
$$

for all even locally constant $\mathbb{Z}_{(p)}$-valued functions $\varepsilon_{L}$ on $H_{S}$ satisfying $\varepsilon_{L}^{\sigma}=$ $\varepsilon_{L}(\forall \sigma \in \Sigma)$ with $\varepsilon_{L}^{\sigma}(h)=\varepsilon_{L}\left(h^{\sigma^{-1}}\right)$.

Proof. Look at the coordinates of

$$
\lambda_{h_{L}} \text { and } \operatorname{ver}\left(\lambda_{g_{K}}\right) \text { in } \mathbb{Z}_{p}\left[H_{S} / V\right] / p^{m_{L}(V)-1}
$$

for a $\Sigma$-stable admissible open subgroup $V \leq H_{S}$ containing the group $C$ generated by all elements $c_{w}$. Note that $\operatorname{ver}\left(\lambda_{g_{K}}\right)$ is then the image under 'ver' of the $U$-coordinate of $\lambda_{g_{K}}$, where $U=\operatorname{ver}^{-1}(V) \leq G_{S}$ contains $N$. These coordinates are the images of

(i) $\sum_{y \in H_{S} / V} \Delta_{h_{L}}\left(1-k, \delta_{L}^{(y)}\right) \mathcal{N}_{L}(y)^{-k} y$, respectively

(ii) $\sum_{x \in G_{S} / U} \Delta_{g_{K}}\left(1-p k, \delta_{K}^{(x)}\right) \mathcal{N}_{K}(x)^{-p k} \operatorname{ver}(x)$

in $\left(\mathbb{Z}_{p}\left[H_{S} / V\right] / p^{m_{L}(V)-1}\right)^{\Sigma}$ by Proposition 2 (recall that it asserts independence of $\lambda_{g}$ from $k$ ).

We show that the sums in (i),(ii) are congruent modulo $T(V)$, where $T(V)$ is the $\Sigma$-trace ideal in $\left(\mathbb{Z}_{p}\left[H_{S} / V\right] / p^{m_{L}(V)-1}\right)^{\Sigma}$, by distinguishing two cases :

(1) $y$ is fixed by $\Sigma$. Then $\delta_{L}^{(y)}$ is an $\varepsilon_{L}$ as appearing in the proposition and so $\Delta_{h_{L}}\left(1-k, \delta_{L}^{(y)}\right) \equiv \Delta_{g_{K}}\left(1-p k, \delta_{L}^{(y)} \circ \operatorname{ver}\right) \bmod p$. Now, if $y=\operatorname{ver}(x)$, then, because ver : $U / N \rightarrow V$ is an isomorphism, $x$ is uniquely determined by $y$ and $\mathcal{N}_{L}(y)^{-k}=\mathcal{N}_{L}(\operatorname{ver}(x))^{-k}=\mathcal{N}_{K}(x)^{-p k}$. Moreover, $\delta_{L}^{(y)}$ o ver $=\delta_{K}^{(x)}$. Hence the corresponding summands in (i) and (ii) cancel out modulo $T(V)$, since $p \alpha$ is a $\Sigma$-trace whenever $\alpha$ is $\Sigma$-invariant. However, if $y \notin \mathrm{im}$ (ver), then $\delta_{L}^{(y)}$ o ver $=0$, hence the $y$-summand vanishes modulo $T(V)$.

(2) $y$ is not fixed by $\Sigma$. By 2. of Lemma $3, \Delta_{h_{L}}\left(1-k, \delta_{L}^{(y)}\right)=\Delta_{h_{L}}\left(1-k, \delta_{L}^{\left(y^{\sigma}\right)}\right)$, whence the $\Sigma$-orbit of $y$ yields the sum $\Delta_{h_{L}}\left(1-k, \delta_{L}^{(y)}\right) \mathcal{N}_{L}(y)^{-k} \sum_{\sigma \in \Sigma} y^{\sigma}$ which is in $T(V)$.

Now subtracting type (ii) sums from type (i) sums for all $\Sigma$-stable admissible open $V \geq$ $C$ gives a compatible system of elements in $\lim _{V \geq C} T(V) \subset \lim _{V \geq C} \mathbb{Z}_{p}\left[H_{S} / V\right] / p^{m_{L}(V)-1}$. Set $H_{S}^{+}=H_{S} / C$; so $H_{S}^{+}=G\left(L_{S}^{+} / L\right)$ where $L_{S}^{+}$is the maximal totally real subfield of $L_{S}$. Since $T\left(V_{1}\right) \rightarrow T(V)$ is surjective whenever $V_{1} \leq V$, we get a limit $s^{+} \in T^{+} \subset$ $\mathbb{Z}_{p}\left[\left[H_{S}^{+}\right]\right]$. Thus the proposition follows from

LEMma 5.: Suppose that $s \stackrel{\text { def }}{=} \lambda_{h_{L}}-\operatorname{ver}\left(\lambda_{g_{K}}\right) \in \mathbb{Z}_{p}\left[\left[H_{S}\right]\right]^{\Sigma}$ has image $s^{+}$under $\mathbb{Z}_{p}\left[\left[H_{S}\right]\right] \rightarrow \mathbb{Z}_{p}\left[\left[H_{S}^{+}\right]\right]$in the $\Sigma$-trace ideal $T^{+}$in $\mathbb{Z}_{p}\left[\left[H_{S}^{+}\right]\right]^{\Sigma}$. Then $s \in T$. 
Proof. We know, from [Se2, (3.12)], that the Frobenius elements $c_{v} \in G_{S}$ for the real primes $v$ of $K$ satisfy $c_{v}^{2}=1, c_{v} \lambda_{g_{K}}=\lambda_{g_{K}}$, and that they generate the kernel of $G_{S} \rightarrow G_{S}^{+}$. Put $c_{K}=\prod_{v}\left(1+c_{v}\right)$.

The analogous properties hold for the $c_{w}$ for the real primes $w$ of $L$, and we can form $c_{L}$. Moreover,

$$
c_{L} \equiv \operatorname{ver}\left(c_{K}\right) \bmod T \text {. }
$$

To see this, expand $c_{L}$ in a sum of products of $c_{w}$ 's and consider the $\Sigma$-action on the summands. The sum of each orbit of length $p$ is in $T$ and the products fixed by $\Sigma$ add up to $\operatorname{ver}\left(c_{K}\right)$, because $\operatorname{ver}\left(c_{v}\right)=\prod_{w \mid v} c_{w}$ for every $v$.

Now $s^{+} \in T^{+}$and the surjectivity of $T \rightarrow T^{+}$mean that $s^{+}=t^{+}$for some $t \in T$, hence $s-t$ is in the kernel of $\mathbb{Z}_{p}\left[\left[H_{S}\right]\right] \rightarrow \mathbb{Z}_{p}\left[\left[H_{S}^{+}\right]\right]$which is generated by all $1-c_{w}$ as a $\mathbb{Z}_{p}\left[\left[H_{S}\right]\right]$-module. Then $c_{L}(s-t)=0$, implying $c_{L} s \in T$ because $c_{L} \in \mathbb{Z}_{p}\left[\left[H_{S}\right]\right]^{\Sigma}$ and $T$ is an ideal of $\mathbb{Z}_{p}\left[\left[H_{S}\right]\right]^{\Sigma}$.

Moreover $c_{L} \lambda_{h_{L}}=2^{[L: \mathbb{Q}]} \lambda_{h_{L}}$ and

$$
c_{L} \operatorname{ver}\left(\lambda_{g_{K}}\right) \equiv \operatorname{ver}\left(c_{K}\right) \operatorname{ver}\left(\lambda_{g_{K}}\right)=\operatorname{ver}\left(c_{K} \lambda_{g_{K}}\right)=2^{[K: \mathbb{Q}]} \operatorname{ver}\left(\lambda_{g_{K}}\right) \bmod T .
$$

Since $2^{p} \equiv 2 \bmod p$, it follows that $2^{[K: \mathbb{Q}]} s \equiv c_{L}\left(\lambda_{h_{L}}-\operatorname{ver}\left(\lambda_{g_{K}}\right)\right)=c_{L} s \equiv 0 \bmod T$, from which the lemma follows as $p \in T$ is odd.

\section{3. $q$-EXPANSIONS}

Let $[K: \mathbb{Q}]=r$, let $\mathfrak{f}$ be an integral ideal with all prime factors in $S$, and denote the usual Hilbert upper half plane associated to $K$ by $\mathfrak{H}=\{\tau \in K \otimes \mathbb{C}: \Im(\tau) \gg 0\}$.

If $k$ is an even positive integer, we define, as usual, the action of $\mathrm{GL}^{+}(2, K \otimes \mathbb{R})$ of matrices with totally positive determinant on functions $F: \mathfrak{H} \rightarrow \mathbb{C}$ by

$$
\left(F_{\mid k}\left(\begin{array}{ll}
a & b \\
c & d
\end{array}\right)\right)(\tau)=\mathcal{N}(a d-b c)^{k / 2} \mathcal{N}(c \tau+d)^{-k} F\left(\frac{a \tau+b}{c \tau+d}\right),
$$

with $\mathcal{N}: K \otimes \mathbb{C} \rightarrow \mathbb{C}$ denoting the norm.

Set

$$
\Gamma_{00}(\mathfrak{f})=\left\{\left(\begin{array}{ll}
a & b \\
c & d
\end{array}\right) \in \mathrm{SL}(2, K): a, d \in 1+\mathfrak{f}, b \in \mathfrak{D}^{-1}, c \in \mathfrak{f} \mathfrak{D}\right\}
$$

where $\mathfrak{D}$ is the different of $K$. A Hilbert modular form $F$ of weight $k$ on $\Gamma_{00}(\mathfrak{f})$ is a holomorphic function $\mathfrak{H} \rightarrow \mathbb{C} \quad{ }^{6}$ satisfying $F_{\mid k} M=F$ for all $M \in \Gamma_{00}(\mathfrak{f})$. Denote the space of these by $M_{k}\left(\Gamma_{00}(\mathfrak{f}), \mathbb{C}\right)$ (see $\left.[\mathrm{DR},(5.7)]\right)$. Such an $F$ can be expanded as a Fourier series

$$
c(0)+\sum_{\substack{\mu \in \mathfrak{o}_{K} \\ \mu \gg 0}} c(\mu) q^{\mu} \quad \text { with } \quad q^{\mu}=e^{2 \pi i \operatorname{tr}(\mu \tau) \quad 7,},
$$

called the standard $q$-expansion of $F$, i.e., the $q$-expansion at the cusp $\infty=\frac{1}{0}$.

Lemma 6.: Let $\beta \in \mathfrak{o}_{K}$ be totally positive with $\mathfrak{f} \subset \beta \mathfrak{o}_{K}$. There is a Hecke operator $U_{\beta}$ on $M_{k}\left(\Gamma_{00}(\mathfrak{f}), \mathbb{C}\right)$ so that, if $F \in M_{k}\left(\Gamma_{00}(\mathfrak{f}), \mathbb{C}\right)$ has standard qexpansion as above, then $F_{\mid k} U_{\beta} \in M_{k}\left(\Gamma_{00}(\mathfrak{f}), \mathbb{C}\right)$ has standard q-expansion $c(0)+\sum_{\mu \gg 0} c(\beta \mu) q^{\mu}$.

\footnotetext{
6 and holomorphic at infinity, if $K=\mathbb{Q}$

${ }^{7} \mathfrak{o}_{K}$ is the ring of integers in $K$; from now on $\mu$ will always be in $\mathfrak{o}_{K}$
} 
Following [AL, $\S \S 2,3]$ for the proof, let $B=\left(\begin{array}{cc}\beta & 0 \\ 0 & 1\end{array}\right)$ and set $\Omega=B \Gamma_{00}(\mathfrak{f}) B^{-1} \cap \Gamma_{00}(\mathfrak{f})$. The matrices $S_{\xi}=\left(\begin{array}{ll}1 & \xi \\ 0 & 1\end{array}\right)$, with $\xi$ running through a set of coset representatives of $\beta \mathfrak{D}^{-1}$ in $\mathfrak{D}^{-1}$, satisfy $\Gamma_{00}(\mathfrak{f})=\dot{\bigcup}_{\xi} \Omega S_{\xi}$, because $\mathfrak{f} \subset \beta \mathfrak{o}_{K}$.

Define $U_{\beta}$ on $M_{k}\left(\Gamma_{00}(\mathfrak{f}), \mathbb{C}\right)$ by

$$
F_{\mid k} U_{\beta}=\mathcal{N}(\beta)^{\frac{k}{2}-1} \sum_{\xi} F_{\mid k} B^{-1} S_{\xi}, \text { with } \mathcal{N} \text { as above in this section. }
$$

Then $F_{\mid k} B^{-1}$ is modular on $B \Gamma_{00}(\mathfrak{f}) B^{-1}$, hence on $\Omega$. The usual averaging argument then shows that $F_{\mid k} U_{\beta}$ is modular on $\Gamma_{00}(\mathfrak{f})$. Now,

$$
\begin{aligned}
& \left(F_{\mid k} U_{\beta}\right)(\tau)=\mathcal{N}(\beta)^{-1} \sum_{\xi} F\left(\beta^{-1} \tau+\beta^{-1} \xi\right) \\
& =\mathcal{N}(\beta)^{-1} \sum_{\xi}\left(c(0)+\sum_{\mu \gg 0} c(\mu) e^{2 \pi i \operatorname{tr}\left(\mu \frac{\tau+\xi}{\beta}\right)}\right) \\
& =\mathcal{N}(\beta)^{-1}\left[\mathfrak{D}^{-1}: \beta \mathfrak{D}^{-1}\right] c(0)+\sum_{\mu \gg 0} c(\mu)\left(\mathcal{N}(\beta)^{-1} \sum_{\xi} e^{2 \pi i \operatorname{tr} K / \mathbb{Q}(\mu \xi / \beta)}\right) e^{2 \pi i \operatorname{tr}(\mu \tau / \beta)} \\
& =c(0)+\sum_{\substack{\mu \gg 0 \\
\mu \in \beta \mathfrak{o}_{K}}} c(\mu) e^{2 \pi i \operatorname{tr}(\mu \tau / \beta)}
\end{aligned}
$$

as $\mathcal{N}(\beta)=\left[\mathfrak{D}^{-1}: \beta \mathfrak{D}^{-1}\right]$ and as $\xi \mapsto e^{2 \pi i \operatorname{tr}_{K / \mathbb{Q}}(\mu \xi / \beta)}$ is a character on $\mathfrak{D}^{-1} / \beta \mathfrak{D}^{-1}$. Since $\beta$ is totally positive, the proof of the lemma is complete.

We next discuss restriction of Hilbert modular forms from $L$ to $K$.

The containment $K \subset L$ induces natural maps $\mathfrak{H}_{K} \stackrel{*}{\rightarrow} \mathfrak{H}_{L}$ and $\mathrm{SL}(2, K \otimes \mathbb{R}) \stackrel{*}{\rightarrow} \mathrm{SL}(2, L \otimes$ $\mathbb{R}$ ). For a holomorphic $F: \mathfrak{H}_{L} \rightarrow \mathbb{C}$ define the restriction res $F: \mathfrak{H}_{k} \rightarrow \mathbb{C}$ of $F$ to be the holomorphic function satisfying $(\operatorname{res} F)(\tau)=F\left(\tau^{*}\right)$. Then

$$
(\operatorname{res} F)_{\mid p k} M=\operatorname{res}\left(F_{\mid k} M^{*}\right) \text { for } M \in \mathrm{SL}(2, K \otimes \mathbb{R}) .
$$

The $q$-expansion at a cusp determined by a finite idèle $\alpha \in \hat{K}^{\times}$is discussed in [DR, bottom of p.229 and (5.8)].

Lemma 7.: Let $F \in M_{k}\left(\Gamma_{00}\left(\mathfrak{f o}_{L}\right), \mathbb{C}\right)$ and let $c(0)+\sum_{\substack{\nu \gg 0 \\ \nu \in \mathfrak{o}_{L}}} c(\nu) q_{L}^{\nu}$ be its standard q-expansion (with $\left.q_{L}^{\nu}=e^{2 \pi i \operatorname{tr}_{L}(\nu \tau)}\right)$. Let $\alpha \in \hat{K}^{\times}$. Then

(1) res $F \in M_{p k}\left(\Gamma_{00}(\mathfrak{f}), \mathbb{C}\right)$ has standard $q$-expansion $c(0)+\sum_{\substack{\mu \gg 0 \\ \mu \in \mathfrak{O}_{K}}} c_{*}(\mu) q_{K}^{\mu}$ with $c_{*}(\mu)=\sum_{\substack{\nu \gg 0, \nu \in \mathfrak{o}_{L} \\ \operatorname{tr}_{L / K}(\nu)=\mu}} c(\nu)$ (and $\left.q_{K}^{\mu}=e^{2 \pi i \operatorname{tr}_{K}(\mu \tau)}\right)$,

(2) the constant term of $\operatorname{res} F$ at the cusp determined by $\alpha$ equals the constant term of $F$ at the cusp determined by $\alpha^{*} \in \hat{L}^{\times}$.

Assertion 1. follows from observing that $\operatorname{tr}_{L}\left(\nu \tau^{*}\right)=\operatorname{tr}_{K}\left(\operatorname{tr}_{L / K}(\nu) \tau\right)$ for $\nu \in L, \tau \in$ $K \otimes \mathbb{C}$, and substituting this into the definition.

For 2., the constant terms in question are those of

$$
(\operatorname{res} F)_{\alpha}=(\operatorname{res} F)_{\mid p k}\left(\begin{array}{cc}
\alpha & 0 \\
0 & \alpha^{-1}
\end{array}\right) \text { and } F_{\alpha^{*}}=F_{\mid k}\left(\begin{array}{cc}
\alpha & 0 \\
0 & \alpha^{-1}
\end{array}\right)^{*},
$$

respectively, by [DR, p.229]. By 1., $F_{\alpha^{*}}$ and res $F_{\alpha^{*}}$ have the same constant term in their respective standard $q$-expansion, so it suffices to show $(\operatorname{res} F)_{\alpha}=\operatorname{res} F_{\alpha^{*}}$. For that, decompose $M=\left(\begin{array}{cc}\alpha & 0 \\ 0 & \alpha^{-1}\end{array}\right) \in \mathrm{SL}(2, \hat{K})$ as $M=M_{1} M_{2}$ according to $\mathrm{SL}(2, \hat{K})=$ 
$\widehat{\Gamma_{00}(\mathfrak{f})} \cdot \operatorname{SL}(2, K)$, hence $M^{*}=M_{1}^{*} M_{2}^{*}$ according to $\mathrm{SL}(2, \hat{L})=\widehat{\Gamma_{00}\left(\mathfrak{f o}_{L}\right)} \cdot \mathrm{SL}(2, L)$.

Then

$$
(\operatorname{res} F)_{\alpha}=(\operatorname{res} F)_{\mid p k} M=(\operatorname{res} F)_{\mid p k} M_{2} \stackrel{(\star)}{=} \operatorname{res}\left(F_{\mid k} M_{2}^{*}\right)=\operatorname{res}\left(F_{\mid k} M^{*}\right)=\operatorname{res} F_{\alpha^{*}},
$$

with equation $\stackrel{(\star)}{=}$ referring to the formula displayed prior to Lemma 7 .

\section{Proof of the MAIN RESUlt}

We use the notation of the previous section, except that we now also use $\mathcal{N}$ for the norm map $K \rightarrow \mathbb{Q}$ and any norm map derived from it ${ }^{8}$, as in $[\mathrm{DR}, \S 2]$.

We attach an Eisenstein series of every even weight $k$ to even locally constant $\mathbb{C}$-valued functions $\varepsilon$ via [DR, (6.1)].

Proposition 8.: Let $\varepsilon$ be an even locally constant $\mathbb{C}$-valued function on $G_{S}$.

(1) There is an integral ideal $\mathfrak{f}$ in $K$ with all its prime factors in $S$ and a modular form $G_{k, \varepsilon} \in M_{k}\left(\Gamma_{00}(\mathfrak{f}), \mathbb{C}\right)$ with standard q-expansion

$$
2^{-r} \zeta_{K}(1-k, \varepsilon)+\sum_{\substack{\mu \gg 0 \\ \mu \in \mathfrak{o}_{K}}}\left(\sum_{\substack{\mu \in \mathfrak{a} \subset \mathfrak{o}_{K} \\ \mathfrak{a} \text { prime to } S}} \varepsilon(\mathfrak{a}) \mathcal{N}(\mathfrak{a})^{k-1}\right) q^{\mu}
$$

where $\varepsilon(\mathfrak{a})=\varepsilon\left(g_{\mathfrak{a}}\right)$ with $g_{\mathfrak{a}} \in G_{S}$ the Artin symbol of $\mathfrak{a}$.

(2) Its q-expansion at the cusp determined by $\alpha \in \hat{K}^{\times}$has constant term

$$
\mathcal{N}((\alpha))^{k} 2^{-r} \zeta_{K}\left(1-k, \varepsilon_{a}\right),
$$

where $(\alpha)$ is the ideal generated by $\alpha$ and $a \in G_{S}$ is the image of $\alpha$ under the map

$$
\hat{K}^{\times} \stackrel{j}{\longrightarrow} G=G\left(K^{\mathrm{ab}} / K\right) \rightarrow G_{S}
$$

with $j$ taken from $\left[D R\right.$, (2.22)] and the identification $G=G\left(K^{\mathrm{ab}} / K\right)$ as in [DR, p.240], via the Artin symbol on integral ideals prime to $\mathfrak{f}$.

(3) $\mathcal{N}((\alpha))=\mathcal{N}\left(\alpha_{p}\right) \cdot \mathcal{N}_{p}(a)$ where $\alpha_{p} \in K \otimes \mathbb{Q}_{p}$ is the p-component of $\alpha \in \hat{K}^{\times}$and $\mathcal{N}_{p}(a)=\mathcal{N}_{K, p}(a)$, as in $\S 1$.

For 1. choose an open subgroup $U$ of $G_{S}$ so that $\varepsilon$ is constant on each coset of $G_{S} / U$. Let $\mathfrak{f}$ be an integral ideal which is a multiple of the conductor of the field fixed by $U$ acting on $K_{S}$ and with all its prime factors in $S$. Then the Artin symbol maps the strict ideal class group $G_{f}$ onto $G_{S} / U$. Viewing $G_{f}$ as the group of invertible elements of $A_{\mathfrak{f}}$, as in [DR, (2.6)], makes $\varepsilon$ a map on $G=\lim _{f^{\prime} \subset f} G_{f^{\prime}}$. Finally extend $\varepsilon$ to $I$ by zero 9. In particular, if $\mathfrak{a}$ is an integral ideal prime to $S$, then $\varepsilon(\mathfrak{a})=\varepsilon\left(g_{\mathfrak{a}}\right)$. Moreover, by [DR, (2.3) and (2.4)], $\varepsilon(\mathfrak{a})=0$ for every (fractional) ideal $\mathfrak{a}$ of $K$ which is not integral and prime to $S$.

Now, with this $\varepsilon,[\mathrm{DR},(6.2)]$ gives the standard $q$-expansion of $G_{k, \varepsilon}$ :

$$
2^{-r} \zeta_{K}(1-k, \varepsilon)+\sum_{\substack{\mu \gg 0 \\ \mu \in \mathfrak{o}_{K}}}\left(\sum_{\mathfrak{x} \subset \mathfrak{o}_{K}} \varepsilon\left(\mu \mathfrak{x}^{-1}\right) \mathcal{N}\left(\mu \mathfrak{x}^{-1}\right)^{k-1}\right) q^{\mu},
$$

\footnotetext{
${ }^{8}$ hence consistent with our usage in $\S 3$

${ }^{9}$ for the definition of $I$ see $[\mathrm{DR}, \S 2]$
} 
where we have chosen the ideal $\mathfrak{B}$ of $[\mathrm{DR}]$ to be $\mathfrak{o}_{K}$. Set $\mathfrak{a}=\mu \mathfrak{x}^{-1}$, so $\mu \in \mathfrak{a}$, and we may assume that $\mathfrak{a}$ is integral and prime to $S$, because otherwise $\varepsilon$ will be zero on $\mathfrak{a}$. Thus the above $\mu$ th coefficient is turned into $\sum_{\substack{\mu \in \mathfrak{a} \subset \mathfrak{o}_{K} \\ \text { primeto } S}} \varepsilon(\mathfrak{a}) \mathcal{N}(\mathfrak{a})^{k-1}$.

For 2., [DR, (6.2)] shows that $\mathcal{N}((\alpha))^{k} 2^{-r} \zeta_{K}\left(1-k, \varepsilon_{c}\right)$ is the constant term of the $q$-expansion at the cusp determined by $\alpha \in \hat{K}^{\times}$, with $c=j(\alpha)$. Our extension of $\varepsilon$ to $G$ has been such that, for $g \in G, \varepsilon_{c}(g)=\varepsilon(c g)=\varepsilon(\overline{c g})=\varepsilon_{\bar{c}}(\bar{g})$ with $\bar{c}, \bar{g}$ the images of $c, g$ in $\underset{f^{\prime} \subset \mathfrak{f}, \text { in } S}{\lim } G_{\mathfrak{f}^{\prime}}$, where ' $\mathfrak{f}^{\prime}$ in $S$ ' means that every prime factor of $\mathfrak{f}^{\prime}$ is in $S$. Hence the commutative square

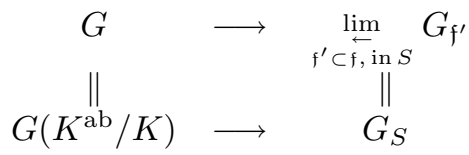

shows $\bar{c}=a$, up to identification.

For 3., we get from [DR, (2.12),(2.16)] that the norm of $c=j(\alpha)$ is $\mathcal{N}(\alpha)^{-1} \mathcal{N}((\alpha))$. Thus the $p$-component of $\mathcal{N}(c) \in \hat{\mathbb{Z}}^{\times}$in $\mathbb{Z}_{p}{ }^{\times}$is $\mathcal{N}\left(\alpha_{p}\right)^{-1} \mathcal{N}((\alpha))$ since $\mathcal{N}((\alpha)) \in \mathbb{Q}^{\times}$. On the other hand, the $p$-component of $\mathcal{N}(c)$ is $\mathcal{N}_{p}(a)$ by the commutative diagram

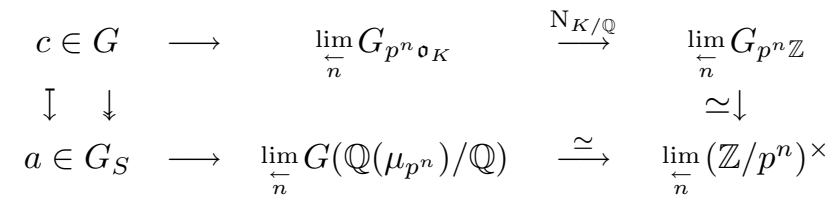

with the left map as in (2a) and $\mu_{p^{n}}$ the $p^{n}$ th roots of unity. Here, the map $G \rightarrow$ $\underset{n}{\lim _{\leftarrow}}\left(\mathbb{Z} / p^{n}\right)^{\times}=\mathbb{Z}_{p}{ }^{\times}$around the top row takes $c$ to the $p$-component of $\mathcal{N}(c)$, which thus is $\mathcal{N}_{p}(a)$.

The proof of the proposition is complete.

LEMMA 9.: ${ }^{10}$ Let $k$ be an even positive integer and $\varepsilon_{L}$ an even locally constant $\mathbb{Z}_{(p)}$-valued function on $H_{S}$. There is an integral ideal $\mathfrak{f} \subset p \mathfrak{o}_{K}$ with all prime factors in $S$, so that

$$
E=\left(\operatorname{res} G_{k, \varepsilon_{L}}\right)_{\mid p k} U_{p}-G_{p k, \varepsilon_{L} \text { over }} \text { is in } M_{p k}\left(\Gamma_{00}(\mathfrak{f}), \mathbb{C}\right){ }^{11} .
$$

If $\varepsilon_{L}^{\sigma}=\varepsilon_{L}$ for all $\sigma \in \Sigma$, then the constant term of the standard q-expansion of $E$ is

$$
2^{-p r} \zeta_{L}\left(1-k, \varepsilon_{L}\right)-2^{-r} \zeta_{K}\left(1-p k, \varepsilon_{L} \text { o ver }\right)
$$

and all non-constant coefficients are in $p \mathbb{Z}_{(p)}$.

Choose an $\mathfrak{f} \subset p \mathfrak{o}_{K}$ by Proposition 8 so that $G_{p k, \varepsilon_{L} \text { over }} \in M_{p k}\left(\Gamma_{00}(\mathfrak{f}), \mathbb{C}\right)$ and $G_{k, \varepsilon_{L}} \in$ $M_{k}\left(\Gamma_{00}\left(\mathfrak{f o}_{L}\right), \mathbb{C}\right)$. Using Lemmas 6 and 7 , the standard $q$-expansion of $G_{p k, \varepsilon_{L} \text { over }}$ is

$$
2^{-r} \zeta_{K}\left(1-p k, \varepsilon_{L} \circ \text { ver }\right)+\sum_{\substack{\mu \gg 0 \\ \mu \in \mathfrak{o}_{K}}}\left(\sum_{\substack{\mu \in \mathfrak{a} \subset \mathfrak{o}_{K} \\ \mathfrak{a} \text { prime to } S}} \varepsilon_{L}\left(\mathfrak{a o}_{L}\right) \mathcal{N}_{K}(\mathfrak{a})^{p k-1}\right) q_{K}^{\mu}
$$

\footnotetext{
${ }^{10}$ compare [Ty]

$11_{\text {with }} U_{p}$ as in Lemma 6
} 
because $\left(\varepsilon_{L} \circ \operatorname{ver}\right)(\mathfrak{a})=\left(\varepsilon_{L} \circ \operatorname{ver}\right)\left(g_{\mathfrak{a}}\right)=\varepsilon_{L}\left(\operatorname{ver}\left(g_{\mathfrak{a}}\right)\right)=\varepsilon_{L}\left(\mathfrak{a o}_{L}\right)$ (see [Se1, VII, 8$\left.]\right)$, and that of $\left.\left(\operatorname{res} G_{k, \varepsilon_{L}}\right)\right)_{\mid p k} U_{p}$ is

$$
2^{-p r} \zeta_{L}\left(1-k, \varepsilon_{L}\right)+\sum_{\substack{\mu \gg 0 \\ \mu \in \mathfrak{o}_{K}}}\left(\sum_{\substack{(\mathfrak{b}, \nu) \text { so } \nu \in \mathfrak{b}^{\prime} \mathfrak{o}_{L}, \nu \gg 0 \\ \mathfrak{b} \text { prime to } S, \operatorname{tr}_{L / K}(\nu)=p \mu}} \varepsilon_{L}(\mathfrak{b}) \mathcal{N}_{L}(\mathfrak{b})^{k-1}\right) q_{K}^{\mu} .
$$

Hence, the $\mu$ th coefficient of $E$ is

$$
\sum_{(\mathfrak{b}, \nu)} \varepsilon_{L}(\mathfrak{b}) \mathcal{N}_{L}(\mathfrak{b})^{k-1}-\sum_{\mathfrak{a}} \varepsilon_{L}\left(\mathfrak{a} \mathfrak{o}_{L}\right) \mathcal{N}_{K}(\mathfrak{a})^{p k-1}
$$

with $(\mathfrak{b}, \nu)$ so that $\nu \gg 0, \nu \in \mathfrak{b} \subset \mathfrak{o}_{L}, \mathfrak{b}$ prime to $S, \operatorname{tr}_{L / K}(\nu)=p \mu$ and $\mathfrak{a} \subset \mathfrak{o}_{K}$ prime to $S$.

The group $\Sigma$ acts on the pairs $(\mathfrak{b}, \nu)$ by $(\mathfrak{b}, \nu)^{\sigma}=\left(\mathfrak{b}^{\sigma}, \nu^{\sigma}\right)$. If $\Sigma$ moves $(\mathfrak{b}, \nu)$, then the orbit sum $\sum_{\sigma} \varepsilon_{L}\left(\mathfrak{b}^{\sigma}\right) \mathcal{N}_{L}\left(\mathfrak{b}^{\sigma}\right)^{k-1}=p \varepsilon_{L}(\mathfrak{b}) \mathcal{N}_{L}(\mathfrak{b})^{k-1}$ because $\varepsilon_{L}\left(\mathfrak{b}^{\sigma}\right)=\varepsilon_{L}^{\sigma^{-1}}(\mathfrak{b})=$ $\varepsilon_{L}(\mathfrak{b})$ and $\mathcal{N}_{L}\left(\mathfrak{b}^{\sigma}\right)=\mathcal{N}_{L}(\mathfrak{b})$.

However, if $\Sigma$ fixes $(\mathfrak{b}, \nu)$, then $\nu \in K, \operatorname{tr}_{L / K}(\nu)=p \mu$, so $\nu=\mu$, and $\mathfrak{b}^{\sigma}=\mathfrak{b}$, so $\mathfrak{b}=\mathfrak{a o}_{L}$ for a unique integral ideal $\mathfrak{a}$ of $K$ prime to $S$, since $S$ contains all primes which are ramified in $L$. Thus $(\mathfrak{b}, \nu)=\left(\mathfrak{a o}_{L}, \mu\right)$.

The above claim on $E$ now follows from

$$
\begin{aligned}
& \varepsilon_{L}(\mathfrak{b}) \mathcal{N}_{L}(\mathfrak{b})^{k-1}=\varepsilon_{L}\left(\mathfrak{a} \mathfrak{o}_{L}\right) \mathcal{N}_{L}\left(\mathfrak{a} \mathfrak{o}_{L}\right)^{k-1}=\varepsilon_{L}\left(\mathfrak{a} \mathfrak{o}_{L}\right) \mathcal{N}_{K}(\mathfrak{a})^{p(k-1)} \\
& \equiv \varepsilon_{L}\left(\mathfrak{a} \mathfrak{o}_{L}\right) \mathcal{N}_{K}(\mathfrak{a})^{p k-1} \bmod p, \text { by } \mathcal{N}_{K}(\mathfrak{a})^{p-1} \equiv 1 \bmod p
\end{aligned}
$$

We finally turn to the Proof of the Theorem stated in the introduction. We check the sufficient conditions for every $\varepsilon_{L}$ as in Proposition 4 . These are the $\varepsilon_{L}$ appearing in Lemma 9. With $E$ as in Lemma 9 and $\alpha \in \hat{K}^{\times}$, let $E_{\alpha}$ be the $q$-expansion of $E$ at the cusp determined by $\alpha$ and let $E(\alpha)=\mathcal{N}_{K}\left(\alpha_{p}\right)^{-p k} E_{\alpha}$.

Since, by [DR, (2.23)], the map $j$ in $(2 a)$ is surjective, there is an idèle $\gamma \in \hat{K}^{\times}$which maps to $g_{K} \in G_{S}$ by $(2 a)$. According to Lemma $9, E(1)=E_{1}$ has non-constant coefficients in $p \mathbb{Z}_{(p)}$. Then, by [DR, (0.3) and Variant: Forms on $\Gamma_{00}(\mathfrak{f})$ at the end of $\S 5], E(1)-E(\gamma)$ has constant coefficient in $p \mathbb{Z}_{p}$. This coefficient is, by Lemmas $6,7,9$ and Proposition 8,

$$
\begin{aligned}
& 2^{-p r} \zeta_{L}\left(1-k, \varepsilon_{L}\right)-2^{-r} \zeta_{K}\left(1-p k, \varepsilon_{L} \circ \text { ver }\right)- \\
& \mathcal{N}_{K}\left(\gamma_{p}\right)^{-p k} \mathcal{N}_{K}((\gamma))^{p k}\left[2^{-p r} \zeta_{L}\left(1-k,\left(\varepsilon_{L}\right)_{h_{L}}\right)-2^{-r} \zeta_{K}\left(1-p k,\left(\varepsilon_{L} \circ \text { ver }\right)_{g_{K}}\right)\right] \\
&= 2^{-p r}\left[\zeta_{L}\left(1-k, \varepsilon_{L}\right)-\mathcal{N}_{K}\left(g_{K}\right)^{p k} \zeta_{L}\left(1-k,\left(\varepsilon_{L}\right)_{h_{L}}\right)\right]- \\
& 2^{-r}\left[\zeta_{K}\left(1-p k, \varepsilon_{L} \circ \text { ver }\right)-\mathcal{N}_{K}\left(g_{K}\right)^{p k} \zeta_{K}\left(1-p k,\left(\varepsilon_{L} \circ \text { ver }\right)_{g_{K}}\right)\right] \\
&= 2^{-p r} \Delta_{h_{L}}\left(1-k, \varepsilon_{L}\right)-2^{-r} \Delta_{g_{K}}\left(1-p k, \varepsilon_{L} \circ \text { ver }\right) \equiv \\
& 2^{-r}\left(\Delta_{h_{L}}\left(1-k, \varepsilon_{L}\right)-\Delta_{g_{K}}\left(1-p k, \varepsilon_{L} \circ \text { ver }\right)\right) \bmod p
\end{aligned}
$$

where we have used that $\gamma^{*} \in \hat{L}^{\times}$has image $\operatorname{ver}\left(g_{K}\right)=h_{L}$ under the map $(2 a)_{L}$ as well as $\mathcal{N}_{K}\left(g_{K}\right)^{p}=\mathcal{N}_{L}\left(h_{L}\right)$.

Thus, Proposition 4 finishes the proof. 


\section{Aвout $p=2$}

For $p=2$ the theorem needs to be reformulated because of the "extra" 2-adic divisibilities of $[\mathrm{DR}]$. In view of Lemma 8, we define

$$
\tilde{\zeta}_{K, S}(1-k, \varepsilon)=2^{-r} \zeta_{K, S}(1-k, \varepsilon),
$$

whence $\tilde{\Delta}_{g_{K}}(1-k, \varepsilon)=2^{-r} \Delta_{g_{K}}(1-k, \varepsilon)$ takes values in $\mathbb{Z}_{2}$ for $\mathbb{Z}_{2}$-valued $\varepsilon$, since an admissible subgroup never admits conductor (1) (see [Ri, §3]). Hence $\tilde{\lambda}_{g_{K}}=2^{-r} \lambda_{g_{K}}$ is in $\mathbb{Z}_{2}\left[\left[G_{S}\right]\right]$ (by e.g. Proposition 2). Following the proof of the theorem now shows that

$$
\text { the image of } \operatorname{ver}\left(\tilde{\lambda}_{g_{K}}\right)-\tilde{\lambda}_{h_{L}} \text { under } \mathbb{Z}_{2}\left[\left[H_{S}\right]\right] \rightarrow \mathbb{Z}_{2}\left[\left[H_{S}^{+}\right]\right] \text {is in } T^{+},
$$

in the notation of Lemma 5 . But the proof of Lemma 5 does not work anymore. One imagines that the methods of [DR], which gave the extra 2-adic divisibilities in the first place, would also sharpen the conclusion displayed above.

REMARK. Actually, we can do the same modification for odd $p$. The equivariant "main conjecture" of [RW2] is unaffected because $\left[\mathcal{Q} G_{\infty}, 2\right]$ is then in the kernel of $\partial: K_{1}\left(\mathcal{Q}\left(\mathbb{Z}_{p}\left[\left[G_{\infty}\right]\right]\right)\right) \rightarrow K_{0} T\left(\mathbb{Z}_{p}\left[\left[G_{\infty}\right]\right]\right)$ (see equation $(\Im)$ on p. 550 of $\left.[\mathrm{RW} 2]\right)$.

We acknowledge financial support provided by NSERC and the University of Augsburg.

\section{References}

[AL] A.O.L. Atkin and J. Lehner, Hecke Operators on $\Gamma_{0}(m)$. Math. Ann. 185 (1970), 134-160.

[DR] P. Deligne and K. Ribet, Values of abelian L-functions at negative integers over totally real fields. Invent. Math. 59 (1980), 227-286.

[FK] T. Fukaya and K. Kato, A formulation of conjectures on p-adic zeta functions in noncommutative Iwasawa theory. Proceedings of the St. Petersburg Mathematical Society, vol. XII (ed. N.N. Uraltseva), AMS Translations - Series 2, 219 (2006), 1-86.

[K] K. Kato, Iwasawa theory and generalizations. Proc. ICM, Madrid, Spain, 2006; European Math. Soc. (2007), 335-357.

[Ri] K. Ribet, Report on p-adic L-functions over totally real fields. Astèrisque 61 (1979), 177-192.

[RW] J. Ritter and A. Weiss, Non-abelian pseudomeasures and congruences between abelian Iwasawa L-functions. Pure and Applied Mathematics Quarterly 4 (2008), 1085-1106.

[RW2] _ Toward equivariant Iwasawa theory, II. Indagationes Mathematicae 15 (2004), 549572.

[Se1] J.-P. Serre, Corps locaux. Hermann, Paris (1968).

[Se2] - Sur le résidu de la fonction zêta p-adique d'un corps de nombres. C.R.Acad.Sci. Paris 287 (1978), série A, 183-188.

[Ty] M. J. Taylor, Galois module type congruences for values of L-functions. J. LMS 24 (1981), 441-448.

Institut für Mathematik, Universität Augsburg, 86135 Augsburg, Germany

E-mail address: jr@ritter-maths.de

Department of Mathematics, University of Alberta, Edmonton, Canada T6G 2G1

E-mail address: weissa@ualberta.ca 